\title{
An optimal grey based approach based on TOPSIS concepts for supplier selection problem
}

\begin{abstract}
Supplier selection is a multi-attribute decision making (MADM) problem that is affected by quantitative and qualitative factors, some of which may conflict with each other. Since most of the input information is not known accurately, selecting the right suppliers becomes more difficult. Grey theory is one of the new mathematical methods used to analyze systems with uncertain and incomplete information. In this article, we firstly described two previous grey based methods and then, we proposed a new method based on TOPSIS concepts in grey theory to deal with the problem of selecting suppliers. The new method calculates the weighted connection between each of the alternatives sequence and the positive and negative referential sequence to compare the ranking of grey numbers and select the most desirable supplier. Through this article, it is demonstrated that the new method is a good means of evaluation, and it is also more optimal than the two methods.
\end{abstract}

Keyword: Supplier selection; MADM; New grey based method; TOPSIS concepts; Optimal method 